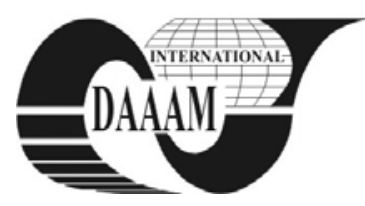

Annals of DAAAM for 2011 \& Proceedings of the 22nd International DAAAM Symposium, Volume 22, No. 1, ISSN 1726-9679 ISBN 978-3-901509-83-4, Editor B. Katalinic, Published by DAAAM International, Vienna, Austria, EU, 2011 Make Harmony between Technology and Nature, and Your Mind will Fly Free as a Bird Annals \& Proceedings of DAAAM International 2011

\title{
FUZZY RISK VERSUS PROBABILISTIC RISK IN PROJECT ECONOMICS
}

\author{
ILOIU, M[irela]; ILOIU, S[orin]; CSIMINGA, D[iana] \& MANGU, S[orin]
}

\begin{abstract}
In this paper we presented the probabilistic risk analysis of the investment projects and we offered a better, more precise alternative - fuzzy risk analysis. This approach, although is analogous to probability theory, can be carried out under weaker assumption and used when limited observational information is available. We proposed here a risk evaluation model based on fuzzy inference in order to determine the risk level for every project risk categories.

Key words: risk, uncertainty, project, fuzzy, inference rule
\end{abstract}

\section{INTRODUCTION}

The paper presents different approaches of the project risk analysis, as a part of the economic analysis of the project. We begin with the defining of the terms "risk" and "uncertainty" and then we present the techniques used for modelling risk quantitatively.

Then we try to argument here than the fuzzy theory offers better results than the probabilistic approach in the project risk analysis because of its ability to facilitate decision-making where there are vague or subjective judgements - the return (or $\mathrm{NPV}$ ) of investment projects.

\section{PROBABILISTIC RISK ANALYSIS IN PROJECT ECONOMICS}

The term "risk and uncertainty" are applied, in most of cases, to the analysis of situations characterized by unknown consequences or outcomes. In essence, risk can be measured, so it is adequate for a quantitative assessment. In a risk situation we can estimate the likelihood of the realized value of a variable falling within stated limits (the variations around the average of a probability calculus). We identify situations of uncertainty when the fluctuations of a variable are such that they cannot be described by a probability calculus.

In practice we define a situation as 'risky' or "uncertain" based on the available information, which defines it as such. All actual outcomes of a project are unknown because they belong to the future (they occur in the future), they can be influenced by many variables and each of these variables may take different values If we have historical or forecast data and we can construct a probability distribution for each variable, the situation is considered and modeled as "risky". If not, we can say we faced to an "uncertain" situation.

In project economic analysis we consider the impacts of unknown outcomes through modeling risk quantitatively. The purpose of quantitative risk analysis is to provide a means of estimating the probability that the project's NPV will fail below zero, or that the project's IRR will fail below the opportunity cost of capital.

The project risk estimating techniques recommend that the results of sensitivity analysis to be used to consider which variable/variables may be appropriate to be the base of a risk analysis (those variables that have a strong influence on project outcomes). After these variables are identified, a number of possible data points are necessary to be specified as well as the frequency (likelihood) of each of these values occurring. (Iloiu \& Iloiu, 2008)

The main purpose of the quantitative risk analysis of a project is to generate a distribution probability function for the project's NPV.

Because risk analysis usually involves the choice of several variables to be varied simultaneously, the project outcomes are subject to more than one source of risk. The mathematical complexity of the model claims computer software in order to make the calculations. That is simulation analysis (or Monte Carlo analysis). It means that values for individual variables are generated randomly according to their respective probability distributions, combined with other randomly-generated values for the other variables, and these figures are used to calculate an estimate of the project NPV. The process is repeated a large number of times (a number specified by the analyst) and an average (the expected NPV) is produced together with an associated probability distribution.

There are many techniques for risk modeling, but more and more experts consider them less precise for making risk estimations. Fuzzy representation may be a possible solution to obtain realistic results in project risk assessment, of course, in certain limits.

\section{FUZZY RISK ANALYSIS - A BETTER ALTERNATIVE}

We do not say here that fuzzy logic can replace robust statistical methods in measuring project's risk, but it can certainly provide a more rigorous framework than the probabilistic assessment. Fuzzy logic is not in competition with mathematical probability theory in the process of evaluating random events. It can be viewed as a complement to probability theory for dealing with real problems in which the available information is subjective, incomplete or unreliable, as may be the case of many investment projects.

Fuzzy set theory is a branch of mathematics introduced in 1965 by Lofti Zadeh in the paper Fuzzy Sets. The paper applied multivalued logic to sets or groups of objects and introduced the concept of "intermediate" membership of a set. In fact this is an advance on the binary logic of classical set theory, where an element is either a full member of a set or not. A framework can be established, on this basis, in which the concept of multivalence or "vagueness" can be managed using methods analogous to those used in classical set theory. (Iloiu \& Csiminga, 2007)

The concept of intermediate membership states that everything is a matter of degree and it helps us to work with characteristics that exists in real world and are characterized by multivalence rather than by bivalence.

We can say that an important feature of fuzzy logic is its ability to facilitate decision-making where there are vague or subjective judgements as inputs to the decision process. 
In most of cases in real world, the available data are insufficient to permit estimating reliably the frequencies of release of risk factors or other characteristics of concern.

There are many models for fuzzy risk analysis. The oldest approach to fuzzy risk analysis is based on the premise that one can provide the input of natural language estimate as probability of failure or loss (negative outcomes or NPV). It can be called combining model where the main work is to combine the fuzzy risks of subsystems to from the fuzzy risk of the entire system. In fact, the probabilities can be represented by natural language or fuzzy numbers.

For dealing with decision-making problems in which the decision-maker has a vague and incomplete information about results and external factors, basic decision rules, based on fuzzy risk intervals, are developed by Delgado, Verdegay and Vila. These fuzzy models open ways for computerization of the process of risk analysis, using a variety of classical fuzzy techniques.

The aim of risk analysis is to describe or to understand some states of a system serving for risk management to reduce or control loss. The states are relative to adverse outcomes, that is why it is necessary for risk analysis to show the relations among states, time, inputs and so on. A probability distribution is just a relation between events and probabilities of occurrences, where an event and a probability value can be regarded as a state and an input, respectively. In many systems (technical, economical or social) it is impossible to precisely calculate the relation, so we deal with the problem of imprecise probability. In fact, the aim of risk analysis is to answer how and why an adverse outcome produces. The risk essence is dynamics of adverse outcomes

Many times it is difficult to obtain the state equations and all data we need and it may be unnecessary to study the equations over.

Risk is a complex phenomenon, especially related to economic processes. When the complex nature is ignored, concept of risk may reduced to be probability-risk, which means that we can find a possibility distribution, obeying a statistical law, to show the risk phenomenon properly. As stated before, it is impossible to obtain a precise probability estimation in many cases. We have to note, here, that sometimes in literature the word probability is used in cases that are clearly possibilistic.

Fuzzy risk can be defined as an approximate representation to show risk with fuzzy theory and techniques. In fact, a fuzzy risk is a fuzzy relation between loss events (negative outcomes of a project) and concerning factors. (http://adrem.org.cn/CEE category html, 2011)

We propose in this paper a possible model for a fuzzy-risk analysis of an investment project. We constructed a model which has two input variables: probability of occurrence for a type of project risk (P) with three levels - low(L), medium (M) and high $(\mathrm{H})$ (the fuzzy set) and the impact of that type of risk on the value of project outcomes (or NPV), noted I, with the same three levels (low, medium, high).. The output variable of the model is the risk level for the considered type of risk (R). Its fuzzy set is: very low (VL), low (L), medium (M), high (H) and very high $(\mathrm{VH})$. The risk evaluation model consists from a set of predefined rules for establishing risk level in investment projects. These inference rules are mentioned in Table 1. (Iloiu, 2005)

\begin{tabular}{|c|c|c|c|}
\hline $\mathrm{P} / \mathrm{I}$ & $\mathrm{L}$ & $\mathrm{M}$ & $\mathrm{H}$ \\
\hline $\mathrm{L}$ & $\mathrm{VL}$ & $\mathrm{L}$ & $\mathrm{L}$ \\
\hline $\mathrm{M}$ & $\mathrm{L}$ & $\mathrm{M}$ & $\mathrm{H}$ \\
\hline $\mathrm{H}$ & $\mathrm{M}$ & $\mathrm{H}$ & $\mathrm{VH}$ \\
\hline
\end{tabular}

Tab.1 The inference rules of the model

The proposed model offers only a quantitative valuation of risk because the decision of accepting/rejection of the project is taken by the human agent. In conclusion, the output of the proposed model is not a form of decision, but an important parameter to make a proper decision. Then, in the process of defuzzification, the final fuzzy set is decomposed in simple shapes: triangles, rectangles and trapezes. Finally we can calculate the risk level.

The model illustrates an alternative approach of the complexity and imprecision that arise in projects risk evaluation, with more fidelity to the type risks involved in this particular problem.

But we must recognize the potential limitation of fuzzy logic for expressing project risk. One problem is the strong reliance on subjective inputs - fuzzy methods might provide more opportunity to abuse the use of subjective inputs. Second, although well suited to addressing uncertainty (lack of knowledge) some argue that for addressing variability, fuzzy logic has not been shown as superior to standard statistical descriptions. Because it can fail to capture the value range of complex data sets and the correlation among parameters, fuzzy arithmetic may not be appropriate for routine use in risk assessments concerned primarily with variability.

\section{CONCLUSIONS}

The available techniques for risk modelling on the basis of probability distributions are considered as standard practice in project risk analysis. Sensitivity analysis and Monte Carlo simulation are well-known.

Although probabilistic assessments based on tools such as Monte Carlo are analogous to assessments based on fuzzy logic, these two methods differ significantly both in approach and interpretation of results. One key advantage of fuzzy logic over Monte Carlo method is the ability to confront linguistic variables. With Monte Carlo methods, we must often force continuous distribution to fit linguistic variables for probabilistic assessments. Fuzzy techniques combines outcomes from different sets in a way that is analogous to but different from Monte Carlo methods. Possibility theory can be used as an alternative to probabilistic analysis, but this creates the potential for interpreting membership functions as probability distributions.

Risk assessment is currently based on the implicit premise that probability theory provides the necessary and sufficient tools for dealing with risk and variability. The key advantage of fuzzy methods is the way they reflect the human mind in its remarkable ability to store and process information which is consistently imprecise, uncertain and resistant to classification. Our model illustrates the ability of fuzzy logic to integrate statistical measurements with imprecision of parameter. We claim that fuzzy logic and probability theory are complementary and not competitive.

\section{REFERENCES}

Delgado, M., Verdegay J.L., Villa M.A., A model for linguistic partial information in decision-making problems, International Journal of Intelligent Systems, 9 (1994)

Huang, C.S.P. (2011). Fuzzy Risk and Calculations, Available from: http://adrem.org.cn/CEE category html.

Iloiu, M. (2005). Riscul in investitiile din industria miniera, Teza de doctorat, Universitatea din Petrosani

Iloiu, M. \& Csiminga, D. (2007). Strategic Risk, Uncertainty and Fuzziness, Acta Universitatis Cibiniensis, Technical Series, vol.LIV, “Lucian Blaga” University of Sibiu, ISSN 1583-7149

Iloiu, M. \& Iloiu, S. (2008). Concept of Probability in Risk Measurements, Annals of the University of Oradea, Fascicle of Textiles-Leatherwork, vol.IX, ISSN 1582-5590

*** (2011) http://www.adb.org - Technical Approaches to the Analysis of Risk in Project Economics 